\title{
Prevalence of Iatrogenic Hyperthyroidism in a Community Hospital
}

\author{
Jay G. Watsky, MD, and Mark A. Koeniger, $M D$
}

Background: There is wide agreement that thyroid hormone replacement should not be given in doses sufficient to suppress thyroid-stimulating hormone (TSH). The purpose of our study was to determine the prevalence of patients currently taking levothyroxine who have an inappropriately suppressed TSH and are thereby at risk for complications of overt or subclinical hyperthyroidism. Those complications of particular concern are osteoporosis and cardiac toxicity, specifically atrial dysrhythmias and development of left ventricular hypertrophy.

Methods: The charts of all 1652 patients dispensed levothyroxine in 1994 from an Air Force community hospital were selected for review. Of these, 905 patients had an available TSH measurement for the same calendar year, and their status was evaluated further.

Results: Of 905 patients, 110 (12.2 percent) had TSH levels of less than $0.1 \mu \mathrm{U} / \mathrm{mL} ; 63$ ( 7 percent) had no indication for suppression, and 21 ( 2.5 percent) were overtly thyrotoxic. The odds ratio for inappropriate suppression in women was 2.83 ( 95 percent $\mathrm{Cl} 1.05-7.68)$.

Conclusion: The frequent use of levothyroxine suggests that TSH suppression could represent a serious public health concern, particularly for postmenopausal women and patients with heart disease. (J Am Board Fam Pract 1998.11:175-9.)

Thyroid hormone preparations account for approximately 1.3 percent of all annual prescriptions and are taken by an estimated 1.8 percent of the US population. ${ }^{1}$ They are extremely safe when used in accordance with recommended guidelines. ${ }^{2,3}$ Overtreatment, however, resulting in a markedly suppressed thyroid-stimulating hormone (TSH $<0.1 \mu \mathrm{U} / \mathrm{mL}$ ), has been shown to lead to osteoporosis and cardiac toxicity.

Endogenous hyperthyroidism is known to have adverse effects on bone mineral content, but only in the past 12 years has exogenous hyperthyroidism been implicated as a risk factor for osteoporosis. ${ }^{4}$ Although a few studies have reported no decrease in bone density with suppressive therapy, ${ }^{5,6}$ the weight of evidence supports the assertion that suppressive therapy contributes to bone loss. ${ }^{7-12}$ Nevertheless, there has been no increase in fracture rates in patients with decreased bone density associated with suppressive therapy. ${ }^{13,14}$ This finding is surprising, because endogenous thyrotoxicosis has been found to increase the rate

Submitted, revised, 17 Sept 1997.

From the 96th Medical Operations Squadron, Eglin Air Force Base, Fla (JGW, MAK). Address reprint requests to Jay Watsky, MD, The Endocrine Group, 4 Executive Park Dr, Albany NY 12203. of hip fracture. ${ }^{14,15}$ Furthermore, the correlation between low bone mass and fracture occurrence is well documented and incontrovertible. ${ }^{16}$ Any factor that contributes to osteoporosis and the subsequent development of nearly 600,000 vertebral compression fractures and 300,000 hip fractures annually, costing in excess of $\$ 10$ billion in the United States alone, ${ }^{17}$ must be considered important and avoided whenever possible.

Clinical experience and multiple clinical investigations suggest that the heart, too, is particularly sensitive to the effects of excess thyroid hormone. ${ }^{18-21}$ Patients with heart disease are at greatest risk for complications from overtreatment. Biondi et $\mathrm{al}^{18}$ found that patients with subclinical hyperthyroidism had marked left ventricular hypertrophy, a known independent predictor of morbidity and mortality. ${ }^{22} \mathrm{~A}$ report appearing in 1979 found that 10 percent of patients with idiopathic atrial fibrillation had subclinical hyperthyroidism. ${ }^{19}$ More recently, based on data extracted from the Framingham Study, Sawin et al ${ }^{20}$ found that low TSH levels correlated with a threefold increased risk of atrial fibrillation after correction for age, smoking, diabetes, hypertension, left ventricular hypertrophy, myocardial infarction, congestive heart failure, and heart murmur. In sum- 
mary, even the mildest degree of hyperthyroidism, as in subclinical hyperthyroidism, might be associated with abnormal cardiac function and clinical heart disease. ${ }^{21}$

Few data are available concerning the extent to which overreplacement with thyroid hormone occurs. Stall et al ${ }^{10}$ found that 10 of 18 women on levothyroxine had their hypothyroidism overtreated based on low TSH values. Of note, these women also had accelerated rates of bone loss. Figge et $\mathrm{al}^{23}$ found 39 of 100 patients with TSH levels less than $0.1 \mu \mathrm{U} / \mathrm{mL}$ on levothyroxine for primary hypothyroidism. Their study was not designed to evaluate the occurrence of inappropriate treatment; they sought to determine the cause of 140 subnormal TSH values associated with normal free thyroxine $\left(T_{4}\right)$ levels observed in 1025 sets of thyroid function test panels ordered on patients referred to their endocrine practice. Thirtytwo patients were undergoing treatment or follow-up of previously diagnosed hyperthyroidism, 25 were on levothyroxine for suppression of benign nodular thyroid disease or goiter, and 13 were on suppression for thyroid malignancy. Twenty-three were on levothyroxine for central hypothyroidism, and the remaining 8 patients had other causes of endogenous thyroid hormone excess. There was no single alternative diagnosis that accounted for a larger proportion of markedly low TSH values with normal free $\mathrm{T}_{4}$ values than excessive treatment with thyroid hormone.

Our study was undertaken to determine the prevalence of inappropriate TSH suppression in patients receiving thyroid hormone, producing unnecessary risk for osteoporosis and cardiac complications. We believe the setting of the study, a community hospital, can provide results more reflective of the use of thyroid hormone in the United States than might be obtained from a study in a major medical center.

\section{Methods}

Data were derived from patients seen at Eglin Air Force Base hospital, an 85-bed facility serving a population of 75,000 in northwest Florida. Fifteen thousand active-duty members receive their medical care at the Air Force hospital, where all their prescriptions are filled and laboratory studies are performed. The remaining 60,000 dependents and retirees have the same services at their choice of an off-base civilian facility or base hospital.
Charts of all patients who received a levothyroxine prescription from the Eglin pharmacy in 1994 and had a TSH measured in the Eglin laboratory during the same year were reviewed. TSH suppression was defined as less than $0.1 \mu \mathrm{U} / \mathrm{mL}$. Documented attention to a suppressed TSH that was not normalized by year-end was defined as "dosage adjustment in progress." Records of all patients with suppressed TSH values were reviewed to determine diagnoses and duration of treatment with levothyroxine. If a patient's record was unavailable for review, patient contact was attempted by telephone. The Mantel-Haenszel estimation was used to determine $P$ values, odds ratios, and confidence intervals, unless otherwise indicated.

The Eglin laboratory used a fluorescent immunoassay (Abbot IMX) to measure TSH and free $\mathrm{T}_{4}$ until July 1994 . TSH normal range was 0.32 to $5.00 \mu \mathrm{U} / \mathrm{mL}$ with a lower limit of detection of $0.029 \mu \mathrm{U} / \mathrm{mL}$. The free $\mathrm{T}_{4}$ normal range was 0.71 to $1.85 \mathrm{ng} / \mathrm{dL}$. A chemiluminescent assay (Sanofi Diagnostics Pasteur) replaced the fluorescent immunoassay in July. The normal range for this TSH assay is 0.49 to $5.66 \mu \mathrm{U} / \mathrm{mL}$ with a lower limit of detection of $0.006 \mu \mathrm{U} / \mathrm{mL}$. The free $\mathrm{T}_{4}$ assay changed as well, with the new normal range at 0.69 to $2.09 \mathrm{ng} / \mathrm{dL}$.

Patients with thyroid cancer or a history of thyroid cancer, as well as patients with nodular thyroid disease or goiter, were grouped as patients for whom suppressive therapy was appropriate (group 1). Patients on replacement thyroid hormone for central hypothyroidism who had markedly subnormal TSH values were also assigned to this group. Patients with suppressed TSH values who were undergoing treatment for primary hypothyroidism or were on thyroid hormone for questionable indications, such as obesity or chronic fatigue, were grouped as patients for whom suppressive therapy was inappropriate (group 2).

\section{Results}

There were 1652 patients who had a levothyroxine prescription filled at the Eglin pharmacy in 1994, and 905 who had their TSH measured at least once in the Eglin laboratory during the same year. Of the 905 patients, 185 (20.4 percent) had a TSH values of less than $0.32 \mu \mathrm{U} / \mathrm{mL}, 110(12.2$ percent) had TSH values suppressed to less than 
Table 1. Diagnoses in 110 Patients Taking Levothyroxine Who Have Markedly Subnormal Thyroid-Stimulating Hormone (TSH) Values $(<0.1 \mu \mathrm{U} / \mathrm{mL})$.

\begin{tabular}{lr}
\hline Diagnosis & Number \\
\hline Appropriate indications for suppression & \\
or low TSH (group 1) & 13 \\
Thyroid cancer & 1 \\
Central hypothyroidism & 11 \\
Goiter & 9 \\
Nodule or adenoma & 34 \\
Total & \\
Inappropriate suppression (group 2) & 57 \\
Hypothyroidism & 6 \\
No documented thyroid disease & 63 \\
Total & 8 \\
No diagnosis & 5 \\
Dosage adjustment in progress & 110 \\
Total &
\end{tabular}

$0.1 \mu \mathrm{U} / \mathrm{mL}$ on at least one occasion, and $116(12.8$ percent) had TSH values greater than 5.66 $\mu \mathrm{U} / \mathrm{mL}$. Chart review or telephone contact established the diagnosis in 102 of 110 patients with markedly suppressed TSH values. Eight patients had no record available and could not be reached by telephone. Five of the 102 patients had a dosage adjustment in progress during the year of study. Of the patients with suppressed TSH values, 34 ( 31 percent) were deemed to have an indication for suppression (group 1), whereas 63 (57 percent) were inappropriately selected for suppressive therapy (group 2) (Table 1).

Overt hyperthyroidism, defined as a free $\mathrm{T}_{4}$ greater than the upper limit of normal for the higher of the two assays $\left(\mathrm{T}_{4}>2.09 \mathrm{ng} / \mathrm{dL}\right)$, was found in 21 of 905 patients, for a prevalence of 2.3 percent. Three of these patients were in group 1 and 18 were in group 2.

Women were disproportionately represented in group 2 when compared with the nonsuppressed group of 842 patients. The odds ratio for inappropriate suppression for women was 2.83 (95 percent CI 1.05 - 7.68). The mean age of the women in group 2 was 51.6 years, which was not different from that of men in either group or women in group 1 . Members of group 2 (those lacking an indication for suppression) were also found to have higher mean free $T_{4}$ levels than those of group 1 (those patients for whom suppressive therapy was appropriate), as displayed in Table 2.
Table 2. Average Free Thyroxine $\left(\mathrm{T}_{4}\right)$ Levels (ng/dL) in Patients With Appropriate Indications for Suppressive Therapy (Group 1, $\mathrm{n}=34$ ) and Patients Lacking Indications for Suppressive Therapy (Group 2, $\mathbf{n}=63$ ).

\begin{tabular}{llll}
\hline Group & Male & Female & Combined \\
\hline Group 1 & 1.27 & 1.66 & 1.58 \\
Group 2 & $1.78(\mathrm{NS})$ & $1.85(\mathrm{NS})$ & $1.84^{*}$ \\
\hline
\end{tabular}

${ }^{*} P<0.007$ group 1 vs group 2 by Kruskal-Wális.

NS - not significant.

\section{Discussion}

Although the optimal degree of suppression in the setting of differentiated thyroid carcinoma is yet to be determined, the usual recommendation is to keep the TSH level at less than $0.1 \mu \mathrm{U} / \mathrm{mL} .^{24.25}$ This conventional practice is challenged by Burmeister $^{26}$ in an extensive review of the literature pertaining to treatment of nonmedullary thyroid carcinoma in which she argues for TSH suppression to 0.1 to $0.4 \mu \mathrm{U} / \mathrm{mL}$. She points out that the benefit of suppressing the TSH to less than $0.1 \mu \mathrm{U} / \mathrm{mL}$ is not established and that the risks of adverse effects on the skeletal and cardiovascular systems are more clear.

Suppressive therapy for benign nodular disease, first suggested by Astwood in 1960, is similarly controversial. Cooper ${ }^{27}$ and Mandel et al ${ }^{28}$ advocate that suppression for benign disease be discontinued after 1 year if not clearly beneficial. Both also argue for particular caution in postmenopausal women and older men. Cooper suggests that these patients be observed for nodule growth for 6 to 12 months and levothyroxine therapy initiated only if the nodule grows. Even in these patients with growing nodules, however, it is believed that suppression of TSH to less than 0.1 $\mu \mathrm{U} / \mathrm{mL}$ is hard to justify.

In this retrospective epidemiologic study of a community in which a military hospital provides a large portion of health care, we found a 12.2 percent prevalence of TSH levels less than 0.1 $\mu \mathrm{U} / \mathrm{mL}$ in patients taking levothyroxine. Of these suppressed patients 57 percent had no indication to warrant suppression. If more stringent criteria were used to define the indications for initiation or continuation of suppressive therapy, as advocated by Burmeister, Cooper, and Mandel et al, the majority of patients in this cohort with inappropriately suppressed TSH values would be much larger.

Women appear to be at particular risk for inap- 
propriate treatment with levothyroxine. Since the average age of the women with suppressed TSH was 51.6 years, many of these women are postmenopausal and therefore at greater risk for suffering both the detrimental bone-thinning and cardiotoxic effects of excessive treatment. The risks of suppressive therapy might be small, but in patients with no indication for such therapy, the risk-benefit ratio is clearly unfavorable.

We were surprised by finding 116 (12.8 percent) of the 905 patients with elevated TSH values. Clearly, those who warrant treatment for hypothyroidism should be given sufficient doses to normalize the TSH, a biochemical hallmark of euthyroidism. This simple and safe intervention often results in patients reporting an improved sense of well-being and can improve cardiac function in those with impaired myocardial contractility. ${ }^{31}$

A strength of this study is that its unique environment provided one large system from which eligible patients in the community can receive medical care at no direct cost. The computerized records of this facility allowed us to evaluate the patterns of pharmacy and laboratory usage of patients in the community cared for by both military and nonmilitary physicians. Similarly, this study environment is a major weakness of the study as well. There were 747 of 1652 patients for whom we have no TSH data during the year. Some of these patients undoubtedly were cared for elsewhere. The majority, we suspect, simply have had inadequate follow-up and could therefore be at increased risk for both undertreatment and overtreatment with thyroid hormone.

Another weakness of the study was the midyear change in methods to evaluate thyroid function and our inability to analyze the data separately. We chose cutoffs in our analysis that would limit the number of outliers. For example, the upper limit of normal for free $\mathrm{T}_{4}$ used to define overt thyrotoxicosis was that of the higher reading assay. The actual prevalence of overt thyrotoxicosis could therefore be substantially higher than 2.5 percent. Similarly, the upper limit of normal for TSH used to define undertreatment of patients with primary hypothyroidism was the higher reading assay. Again, the actual prevalence of subclinical hypothyroidism in patients on replacement thyroid hormone could be considerably higher than 12.8 percent.

In conclusion, our findings suggest that many patients on thyroid hormone therapy are at risk of iatrogenic complications without appropriate indications. Postmenopausal women, who have lost the cardioprotective and bone-preserving benefits of estrogen, are particularly at risk for complications of inappropriate levothyroxine dosing. And yet, women, including those postmenopause, are the patients most likely to be given excessive levothyroxine when suppression is not indicated. Reasons for more frequent overtreatment of women are unclear and open to speculation. Physicians are encouraged to educate patients about the potential toxicity of suppressive therapy and carefully select for this therapy only those most likely to benefit. Further clarification of the risks of thyroid hormone therapy with and without careful monitoring will require further study.

\section{References}

1. Kaufman SC, Gross TP, Kennedy DL. Thyroid hormone use: trends in the United States from 1960 through 1988. Thyroid 1991;1:285-91.

2. Petersen K, Bengtsson C, Lapidus L, Lindstedt G, Nystrom E. Morbidity, mortality, and quality of life for patients treated with levothyroxine. Arch Intern Med 1990;150:2077-81.

3. Burman $\mathrm{KD}$. Is long-term levothyroxine therapy safe? Arch Intern Med 1990;150:2010-3.

4. Fallon MD, Perry HM 3d, Bergfeld M, Droke D, Teitelbaum SL, Avioli LV. Exogenous hyperthyroidism with osteoporosis. Arch Intern Med 1983; 143:442-4.

5. Marcocci C, Golia F, Bruno-Bossio G, Vignali E, Pinchera A. Carefully monitored levothyroxine suppressive therapy is not associated with bone loss in premenopausal women. J Clin Endocrinol Metab 1994;78:818-23.

6. Faber J, Galloe AM. Changes in bone mass during prolonged subclinical hyperthyroidism due to L-thyroxine treatment: a meta-analysis. Eur J Endocrinol 1994;130:350-6.

7. Ross DS, Neer RM, Ridgway EC, Daniels GH. Subclinical hyperthyroidism and reduced bone density as a possible result of prolonged suppression of the pituitary-thyroid axis with L-thyroxine. Am J Med 1987; 82:1167-70.

8. Paul TL, Kerrigan J, Kelly AM, Braverman LE, Baran DT. Long-term L-thyroxine therapy is associated with decreased hip bone density in premenopausal women. JAMA 1988;259:3137-41.

9. Ross DS. Subclinical hyperthyroidism: possible danger of overzealous thyroxine replacement therapy. Mayo Clin Proc 1988;63:1223-9.

10. Stall GM, Harris S, Sokoll LJ, Dawson-Hughes B. Accelerated bone loss in hypothyroid patients 
overtreated with L-thyroxine. Ann Intern Med 1990; 113:265-9.

11. Diamond T, Nery L, Hales I. A therapeutic dilemma: suppressive doses of thyroxine significantly reduce bone mineral measurements in both premenopausal and postmenopausal women with thyroid carcinoma. J Clin Endocrinol Metab 1991;72:1184-8.

12. Adlin EV, Maurer AH, Marks AD, Channick BJ. Bone mineral density in postmenopausal women treated with L-thyroxine. Am J Med 1991;90:360-6.

13. Leese GP, Jung RT, Guthrie C, Waugh N, Browning MC. Morbidity in patients on l-thyroxine: a comparison of those with a normal TSH to those with a suppressed TSH. Clin Endocrinol Oxf 1992;37:500-3.

14. Solomon BL, Wartofsky L, Burman KD. Prevalence of fractures in postmenopausal women with thyroid disease. Thyroid 1993;3:17-23.

15. Bauer DC, Cummings SR, Tao JL, Browner WS. Hyperthyroidism increases the risk of hip fractures: a prospective study. J Bone Miner Res 1992;7(Suppl 1):121.

16. Kanis JA. Osteoporosis. Cambridge, Mass: Blackwell Scientific, 1994:1-20.

17. Consensus Development Conference: diagnosis, prophylaxis, and treatment of osteoporosis. Am J Med 1993;94:646-50.

18. Biondi B, Fazio S, Carella C, Amato G, Cittadini A, Lupoli G, et al. Cardiac effects of long-term thyrotropin suppressive therapy with levothyroxine. J Clin Endocrinol Metab 1993;77:334-8.

19. Forfar JC, Miller HC, Toft AD. Occult thyrotoxicosis: a correctable cause of "ideopathic" atrial fibrillation. Am J Cardiol 1979;44:9-12.

20. Sawin CT, Geller A, Wolf PA, Belanger AJ, Baker E, Bacharach P, et al. Low serum thyrotropin concentrations as a risk factor for atrial fibrillation in older persons. N Engl J Med 1994;331:1249-52.

21. Ladenson PW. Thyrotoxicosis and the heart: something old and something new. J Clin Endocrinol Metab 1993;77:332-3.

22. Levy D, Garrison RJ, Savage DD, Kannel WB. Prognostic implications of echocardiographically determined left ventricular mass in the Framingham Heart Study. N Engl J Med 1990;322:1561-6.

23. Figge J, Leinung $M$, Goodman AD, Izquierdo $R$, Mydosh T, Gates S, et al. The clinical evaluation of patients with subclinical hyperthyroidism and free triiodothyronine (free T3) toxicosis. Am J Med 1994; 96:229-34.

24. Toft AD. Thyroxine therapy. N Engl J Med 1994; 331:174-80.

25. Kaplan MM. Thyroid hormone therapy. what, when, and how much. Postgrad Med 1993;193:249-52, 255 $6,260-2$.

26. Burmeister LA. Thyroid hormone in the treatment of thyroid cancer. Thyroid Today 1994;17(1):1-9.

27. Cooper D. Thyroxine suppressive therapy for benign nodular disease. J Clin Endocrinol Metab 1995;80: 331-4.

28. Mandel SJ, Brent GA, Larsen PR. Levothyroxine therapy in patients with thyroid disease. Ann Intern Med 1993;119:492-502.

29. Wartofsky L. Use of sensitive TSH assay to determine optimal thyroid hormone therapy and avoid osteoporosis. Ann Rev Med 1991;42:341-5.

30. Hamburger JI, Kaplan MM. Hypothyroidism. Don't treat patients who don't have it. Postgrad Med 1989; 86:67-74.

31. Cooper DS, Halpern R, Wood LC, Levin AA, Ridgway EC. L-Thyroxine therapy in subclinical hypothyroidism. A double-blind, placebo-controlled trial. Ann Intern Med 1984;101:18-24. 